\title{
Turn-by-Turn and Bunch-by-Bunch Transverse Profiles of a Single Bunch in a Full Ring
}

\author{
Richard Kraus \\ Office of Science, Summer Undergraduate Laboratory Internship (SULI) \\ University of Nevada, Reno \\ Stanford Linear Accelerator Center \\ Menlo Park, California 94025
}

August 12, 2005

Prepared in partial fulfillment of the requirements of the Office of Science, Department of Energy’s Science Undergraduate Laboratory Internship under the Direction of Dr. Alan S. Fisher in the Accelerator Division of the Stanford Linear Accelerator Center (SLAC).

Participant:

Signature

Research Advisor:

Signature 


\section{TABLE OF CONTENTS}

$\begin{array}{lc}\text { Abstract } & \text { iii } \\ \text { Introduction } & 1 \\ \text { Materials and Methods } & 3 \\ \text { Results } & 7 \\ \text { Discussion } & 8 \\ \text { Acknowledgements } & 10 \\ \text { References } & 10 \\ \text { Tables and Figures } & 11\end{array}$




\begin{abstract}
Turn-by-Turn and Bunch-by-Bunch Transverse Profiles of a Single Bunch in a Full Ring. RICHARD KRAUS (University of Nevada, Reno, NV 89557) ALAN S. FISHER (Stanford Linear Accelerator Center, Menlo Park, CA 94025).
\end{abstract}

The apparatus described in this paper can image the evolution of the transverse profile of a single bunch, isolated from a full PEP-II ring of 1500 bunches. Using this apparatus there are two methods of single bunch imaging; bunch-by-bunch beam profiling can image every bunch in the ring a single bunch at a time with the images of sequential bunches being in order, allowing one to see variations in beam size along a train. Turn-by-turn beam profiling images a single bunch on each successive turn it makes around the ring. This method will be useful in determining the effect that an injected bunch has on a stable bunch as the oscillations of the injected bunch damp out. Turn-by-turn imaging of the synchrotron light uses a system of lenses and mirrors to image many turns of both the major and minor axis of a single bunch across the photocathode of a gateable camera. The bunch-by-bunch method is simpler: because of a focusing mirror used in porting the light from the ring, the synchrotron light from the orbiting electrons becomes an image at a certain distance from the mirror; and since the camera does not use a lens, the photocathode is set exactly at this image distance. Bunch-by-bunch profiling has shown that in the Low Energy Ring (LER) horizontal bunch size decreases along a train. Turn-by-turn profiling has been able to image 100 turns of a single bunch on one exposure of the camera. The turn-by-turn setup has also been able to image 50 turns of the minor axis showing part of the damping process of an oscillating injected charge during a LER fill. The goal is to image the 
damping of oscillations of injected charge for 100 turns of both the major and minor axis throughout the damping process during trickle injection. With some changes to the apparatus this goal is within reach and will make turn-by-turn imaging a very useful tool in beam diagnostics. 


\section{INTRODUCTION}

PEP-II at the Stanford Linear Accelerator Center (SLAC) collides 9-Gev electrons stored in the high-energy ring (HER) with 3.1-Gev positrons stored in the low-energy ring (LER). PEP-II operates with currents of 2.50 A in the LER and 1.5 A in the HER. The collisions create BB-bar meson pairs for the purpose of measuring different decay rates due to charge-parity violation. The frequency of these collisions is dependent upon the luminosity in the rings, which depends both on the current and the transverse beam size. To gain more collisions one could increase the current in the rings, shorten the bunch length, shrink the transverse beam size, or focus the beam more tightly at the IP. However, it is impossible to increase the current in the ring above a certain level because there is not enough RF power available; and even before the current gets to the limit, equipment overheats and starts to malfunction. Shortening the bunch length by using higher RF voltages works except that heating from the increased peak current has caused some beam position monitors to loosen and fall off. And as beams are focused at the interaction point (IP) through a smaller waist the luminosity is limited by the "hourglass effect," where the beams expand more quickly on either side of the focus [1]. The greatest gains in luminosity lie in shrinking the transverse profile of the beam.

Megawatt microwave generators called klystrons accelerate electrons and positrons at PEP-II; there are places in the microwave field where electron or positron bunches can be relatively stable, such places are called RF buckets. Within the HER or LER, bunches travel in trains where every other bucket is filled and each train has between 30 and 70 bunches for a total of typically 1588 bunches in a ring at one time. 
This work images synchrotron light emitted by a single bunch of electrons or positrons onto a gateable intensified charge-coupled device (ICCD) camera. But because an ICCD camera does not have the ability to repeatedly store images at $136 \mathrm{kHz}$, the frequency that a bunch goes around the ring, an apparatus capable of imaging up to one hundred turns of the same bunch using only a few optical components and a gateable ICCD camera is used.

Past work with bunch-by-bunch beam profiling [2] has shown that horizontal beam size decreases with bucket number along a train. We have recently reproduced the experiment and found the same results (see Figure 1). Understanding why the beam decreases in size along the train could give insight into how to decrease the transverse size of every bunch in the train.

During injection, charge from the linear accelerator must be injected from above the PEPII rings at an angle to the path of the stable bunches in the rings. These injected charges are focused towards the axis of the ring by quadrapole magnets causing them to oscillate. These oscillations will eventually damp out because the particles emit synchrotron radiation in their direction of travel but gain energy from the microwave field in the longitudinal direction. A major focus of this experiment is to understand charge injection damping. Questions that should be answered with this research include; what effect does the injected bunch have upon the stable bunch and specifically in the HER why is there greater background at BaBar a few milliseconds after trickle injection when the damping process takes 37.1ms? Imaging an injected bunch during trickle injection is not easy: the injected bunch is $1 / 20^{\text {th }}$ the size of the stable bunch, the bunch will only be visible for the first part of the damping process as after a few milliseconds it will have blended in with the outer part of the stable bunch, and if the tune of the ring is such that the injected bunch is in plane with the stable bunch at the synchrotron light pick-off point, one would not be able to see it at all. 
Alignment is crucial for such an experiment; any major deviation from the layout will cause the image to blur or increase in size such that the image will not fit on the photocathode. The most difficult part about this research will be the analysis of the data, there are 100 images of the major axis and 100 images of the minor axis stored in one frame; simple pixel addition will not suffice.

\section{MATERIALS AND METHODS}

The synchrotron light used in this research comes from a bending dipole magnet on each ring; the light is initially diverted using a water cooled mirror set at grazing incidence with a slit through the center such that the x-rays will pass through to a beam dump and the maximum power on the mirror will be $200 \mathrm{~W} / \mathrm{cm}$. Synchrotron light is usually sent to an interferometer which measures vertical beam size [3]; however, using a button macro in the SLAC Control Panel (SCP) program the light can be diverted using servo-controlled mirrors onto an optical table in building 675 for the HER and 620 for the LER. A diagram of the experimental layout in the LER is shown in Figure 2; the apparatus in the HER uses the same components, the magnifications and beam sizes are different (see Table 1).

The goal of this optical setup is to manipulate the synchrotron light beam to a tall and thin image: thin so that many images will fit across the photocathode and tall so that measurements of the profile can be taken easily. Since the beam is originally an ellipse the major and minor axis measurements are not equal. This requires that the beam be split: to measure both the profile of the major axis and the minor axis (for clarification the beam which images the major axis shall be called the major axis beam and the beam which images the minor 
axis shall be called the minor axis beam). Because there are now two beams on the table, there are four magnifications that need to be set; the major axis $\mathrm{x}$ (horizontal) and $\mathrm{y}$ (vertical) directions, and the minor axis $\mathrm{x}$ and $\mathrm{y}$ directions. Cylindrical lenses are used because they can magnify in one direction and leave the beam unaltered in the other direction. For each axis this allows magnification in one direction to be semi-independent from the other direction; semi because they must both come to a focus on the photocathode of the ICCD camera.

Once on the table the synchrotron light is filtered with a polarizer cube such that only the horizontally polarized light from the emission point continues on the set beampath. This is necessary because synchrotron light is horizontally polarized on the mid-plane of the curve and any vertically polarized light can cause a blurred image. Next the light is filtered with a 30 nanometer wide bandpass filter, centered around 450nm in the LER and 550nm in the HER: this is necessary because lenses have different indices of refraction for different wavelengths and thus if the imaging light has too broad a spectrum the image will never be in focus.

From there the light can be diverted, using a mirror mounted on an insertable ("flipper") mount, to a gated camera for use in Bunch-by-Bunch beam profiling [1], for a sample image see Figure 3. Usually the light will pass over the "flipper" mirror and onto a nominally $50 \%$ beam splitter. Half of the beam will go through an out of plane periscope, which rotates the beam by 90 and brings it to the required height. The beam must be rotated so that both axes of the bunch can be imaged. The major axis beam goes through a cylindrical y-lens (lens that magnifies in the vertical direction) to achieve a slight magnification. The path which images the minor axis is a bit more complicated, to compensate for the additional path length and extra horizontal width of the minor axis beam (the major axis of the particle beam's ellipse) a negative lens must be used to create a smaller virtual image $110 \mathrm{~mm}$ downstream of the true image. Then the minor axis 
beam will go through a magnifying cylindrical y-lens such that the beam's height will expand by a factor of almost three: to use more of the photocathode. At this point the minor axis beam is six millimeters above the major axis beam. Using two, 2-inch mirrors the beams shall be forced parallel above one another and through one last cylindrical $\mathrm{x}$-lens that demagnifies the major axis beam in the $\mathrm{x}$-direction by a factor of 10 and the minor axis beam in the $\mathrm{x}$-direction by a factor of 20. The major and minor profiles of the beam have been manipulated to thin vertical stripes.

These stripes are reflected off a $14.2 \mathrm{~mm}$ tall by $8.5 \mathrm{~mm}$ wide mirror, attached to a scanning galvanometer, onto the photocathode of the ICCD camera. The purpose of the scanning galvanometer is such that in one exposure of the CCD the micro channel plate can be repeatedly gated so that light from only one bunch can be exposed onto the CCD and with the motion of the mirror the next time that bunch comes around the ring the light will be reflected to a new place on the photocathode.

An external trigger, defined as the injection trigger plus the delay between the trigger and the injected bunch less the time the rotating mirror takes to get into position, starts the rotation of the mirror by triggering an arbitrary waveform generator to begin its waveform; the waveform voltage is directly proportional to the rotation of the mirror. Once the light reflected from the rotating mirror has reached the left side of the photocathode another pulse is sent by a delay generator to both the camera and a second delay generator, signifying the injected bunch is here and the camera should begin gating; the second delay generator sends triggers to the camera every $7.32 \mathrm{~s}$, the period of the ring, so as to image the injected bunch on every turn. The delay generator can also be set to trigger the camera every other turn or every third turn by increasing the delay to integer multiples of $7.32 \mathrm{~s}$. 
There is an injection trigger that is given from the Main Control Center (MCC) every time charge is inserted into the PEP-II rings. Due to transport there is a delay between the time the charge is actually injected into the ring and the time at which the delay generator receives the pulse. Finding the delay is quite tedious. The only optimal time to search for the injection delay is right after a ring abort when the ring is being filled one bunch at a time. However, it only takes about eight injections to fill a bunch, which means that the camera, triggered by the injection, can only be gated eight times during the bunch filling process. The period of the ring is $7.32 \mu$ s and thus each gate images $0.915 \mu \mathrm{s}$. Now the injected bunch is in a $0.915 \mu$ s time frame, which with the next abort can be narrowed down further to a $0.114 \mu \mathrm{s}\left(1 / 8^{\text {th }}\right.$ of $\left.0.915 \mu \mathrm{s}\right)$ time frame, the process is continued till the injected bunch has been confined to a 2.1ns time frame. The delay between the injection trigger and the injected bunch will be known once the delay to the injected bunch is identified.

For the purpose of watching the damping of an injected bunch only the minor axis of the particle beam's ellipse will be imaged, for that is the direction in which the injected charge oscillates. The beamsplitter is removed so that all of the synchrotron light will be used in imaging the minor axis. With the camera only gating the bucket that charge is being injected into, there are only a few things to vary. The section of the damping process which the camera images can be varied by changing the delay to the waveform and the length of the section imaged can be changed by having the camera postpone the gates for an integer number of turns.

No analysis software has been written yet for the turn-by-turn images. In bunch-bybunch mode analysis is done using software written in C; the beams are fit to a gaussian profile. 


\section{Results}

Figure 4 shows 94 turns of a single bunch in the LER. The major axis is seen above the minor axis in the picture because the camera inverts the true image. Although the initial goal of this project was to see the largest number of turns possible, images are useless when one image cannot be distinguished from the next. For our present focusing abilities 100 turns seems to be about the limit in resolving one turn from the next in the major axis beam (see Figure 4). As one moves down to 60 turns, each turn is easily resolvable in both the major and minor axis as seen in Figure 5. And if there needed to be absolutely no question as to which photon belonged to which turn, 41 turns across the photocathode are quite well spaced, as seen in Figure 6.

In the LER a section of the damping process for an injected bunch was imaged during a ring fill after an abort. Figure 7 shows 50 sequential turns of an injected bunch oscillating around an empty bucket at the beginning of the filling process. Figure 8 shows another injected bunch oscillating around a small stable bunch in the LER. Figure 9 shows 50 sequential turns of an injected bunch oscillating about a stable bunch that is $1 / 3^{\text {rd }}$ full; these images were taken at the beginning of the LER filling process.

Using the single bunch ring fill method the delay between the injection trigger and the injected bunch is 5649.7ns. For use in bunch-by-bunch mode the delay between the ring turn trigger and bucket zero is 5785.0ns. 


\section{DISCUSSION}

In the LER the minor axis beam does not image nearly as well as the major axis beam, for only at 60 turns does the minor axis beam become resolvable. This was expected for in the LER the minor axis beam started out close to six times as wide as the major axis beam and the negative lens in the minor path only demagnifies the minor axis beam by a factor of two. Thus the image of the minor axis is three times as wide as the image of the major axis. This will not be as great a problem in the HER for the minor axis beam is only three times as wide as the major axis beam.

Magnification and focus are two very important factors in being able to image many turns. This project required multiple changes to the layout in the LER to find magnifications capable of a suitable width and height, such that the major and minor axis beams fit on the photocathode. 100 turns across the photocathode in the LER did not come cheaply, two lenses were liberated from their positions in the HER apparatus for the benefit of the LER. The HER, which has not been completed, will most likely have to go through as many changes in both layout and lens choice. And after many iterations of changing the lenses and the layout it is all for naught without the correct focus: a difference of a millimeter in the position of the camera can mean the difference between 100 turns and 20.

It was only possible to image the injected bunch when all of the light was used in imaging the minor axis of the particle beam ellipse and when the LER was being filled after an abort. It is easier to image the injected bunches at the beginning of a fill because there is no stable charge to block the view of the injected charge. Although imaging injected charge during a fill was a large step, it is very important that charge injected during trickle injection be imaged; 
for that is the charge that affects the colliding beams. Luckily, now that all the delays are set correctly it should be trivial to image the damping process during trickle injection. It is also important to be able to image the major axis of the particle beam ellipse during trickle injection: the oscillating charge should have some very interesting effects on the major axis as well as the minor axis of the particle beam's ellipse.

In order to image the effects of oscillating charge on both axes a greater amount of light will have to get to the camera. Small optics are not passing the full beam and are limiting the total light to the camera; larger, 2-inch optics are on order and will be able to pass most of the beam. A new beam splitter will be used to send the necessary amount of light to each path. Another option is to set the camera to accumulate mode, where images are superimposed on one another to increase the brightness of the image.

This apparatus is quite flexible in the fact that if one wanted to take one clear picture of a single bunch (see Figure 3) they would be able to do so without much effort in bunch-by-bunch mode. Once in turn-by-turn mode, the variations become even simpler: to view more turns all one has to do is lessen the slope of the waveform and increase the number of triggers to the camera. If there was a need to view a single bunch for a long period of time, the delay on the delay generator could be set to wait for any integer number of turns before it triggers the camera.

With the correct magnifications and foci the bunch-by-bunch and turn-by-turn single bunch imaging systems will become useful tools in beam diagnostics. 


\section{ACKNOWLEDGEMENTS}

First and foremost I would like to thank my mentor, Alan S. Fisher, for his guidance and assistance throughout this internship. I have learned a great deal from talking and working with

him. I want to thank Mark Petree for his technical support and Benny Chan for his assistance in this research. I would like to thank Helen Quinn and James Lindesay for their support of the SULI program. I would also like to thank the Department of Energy's Office of Science and the Stanford Linear Accelerator Center for creating, organizing, and funding the SULI program.

\section{RESOURCES}

[1] A.S. Fisher, A. Novokhatski, J.L. Turner, U. Wienands, G. Yocky, R. Holtzaapple, “BunchLength Measurements in PEP-II,” presented at IEEE Particle Accelerator Conference, Knoxville, Tennessee, 2005.

[2] Robert Holtzapple, Denis Dujmic, and Alan S. Fisher, “ Single-Bunch Tune and Beam Size Measurements along Bunch Trains in PEP-II,” presented at IEEE Particle Accelerator Conference, Knoxville, Tennessee, 2005.

[3] A.S. Fisher, M. Petree, E. L. Bong, “A Synchrotron-Light Interferometer for PEP-II,” presented at Beam Instrumentation Workshop, Cambridge, Massachusetts, 2000. 


\section{FIGURES AND DIAGRAMS}

\begin{tabular}{|l|l|l|l|l|r|r|}
\hline HER Magnifications and Dimensions & & & & Major & Minor \\
\hline & & & & & $(\mathrm{mm})$ & $(\mathrm{mm})$ \\
\hline
\end{tabular}

\begin{tabular}{|l|l|l|l|r|r|r|}
\hline LER Magnifications and Dimensions & & & & \multicolumn{1}{c|}{ Major } & \multicolumn{1}{c|}{ Minor } \\
\hline
\end{tabular}

Table 1: Magnifications and image distances for HER and LER.

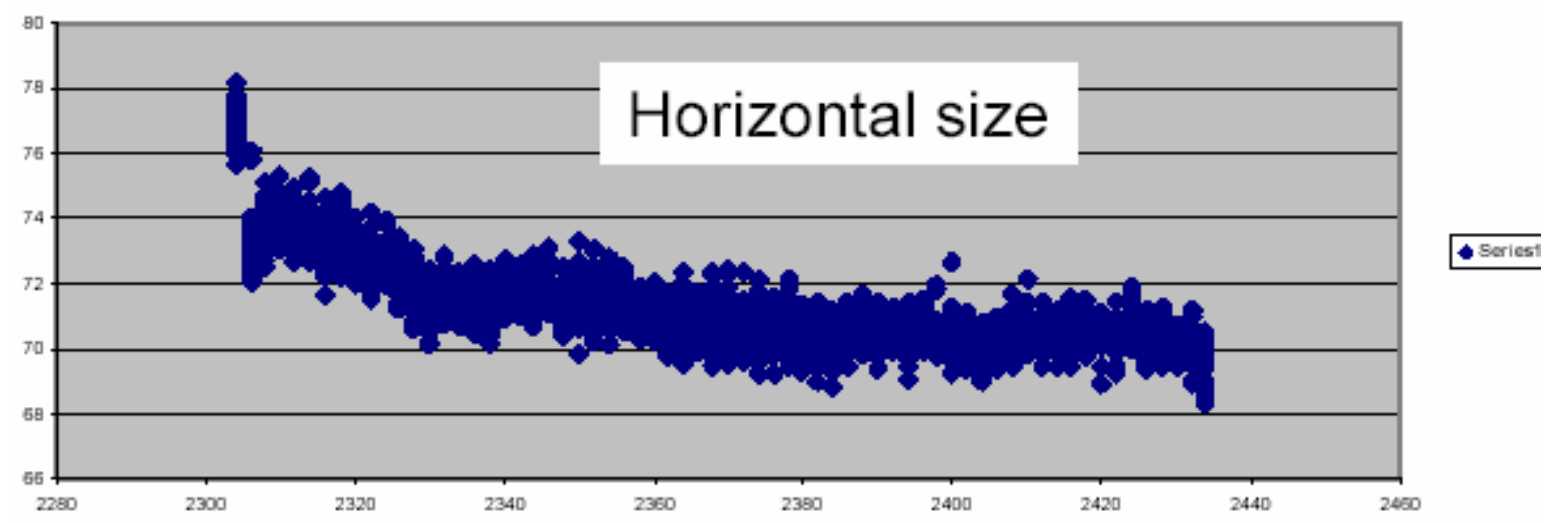

Figure 1: Bunch-by-bunch diagram showing decrease in beam size along bunch train in LER. $\mathrm{X}$-axis shows bunch number, $\mathrm{Y}$-axis in arbitrary units of size. 


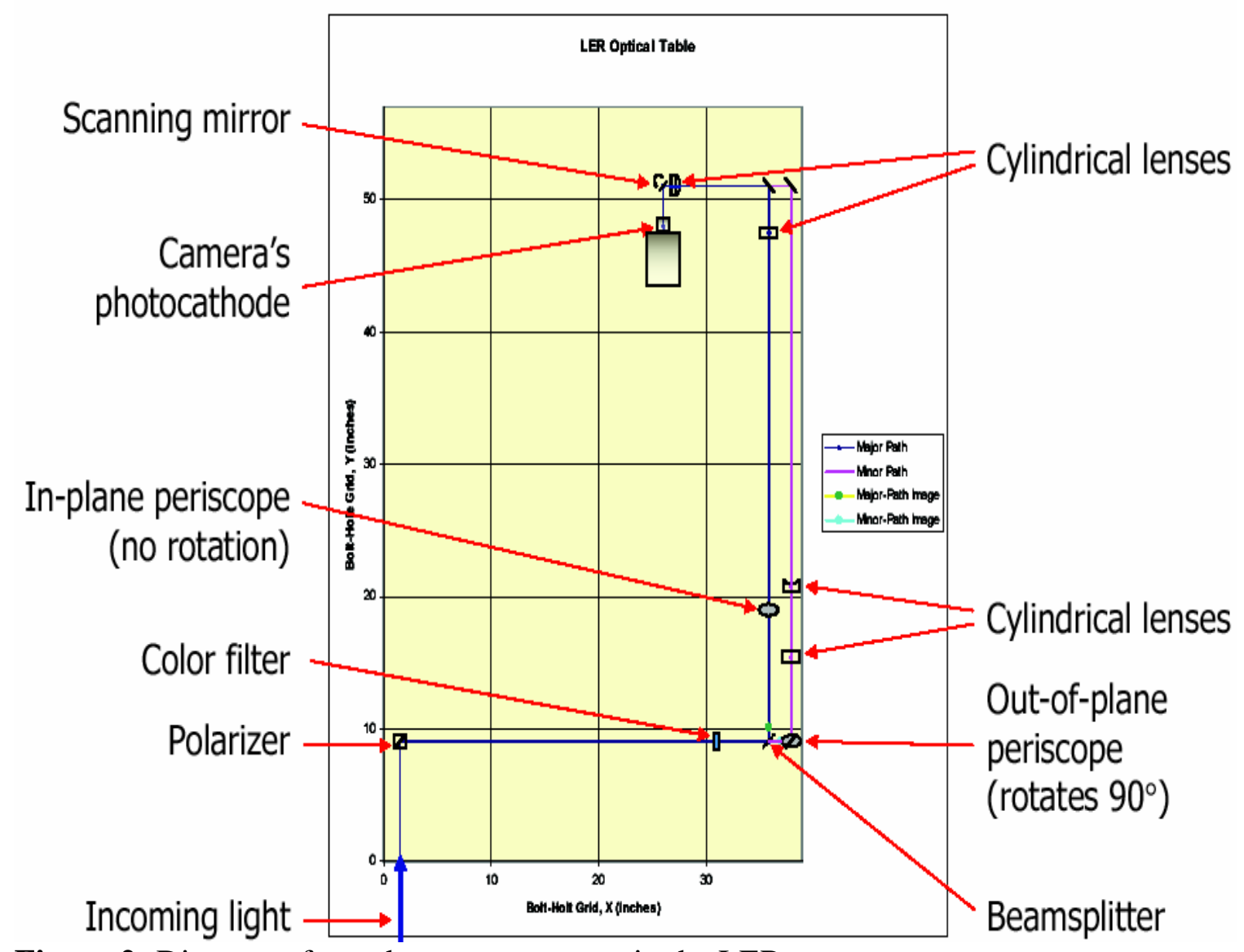

Figure 2: Diagram of turn-by-turn apparatus in the LER

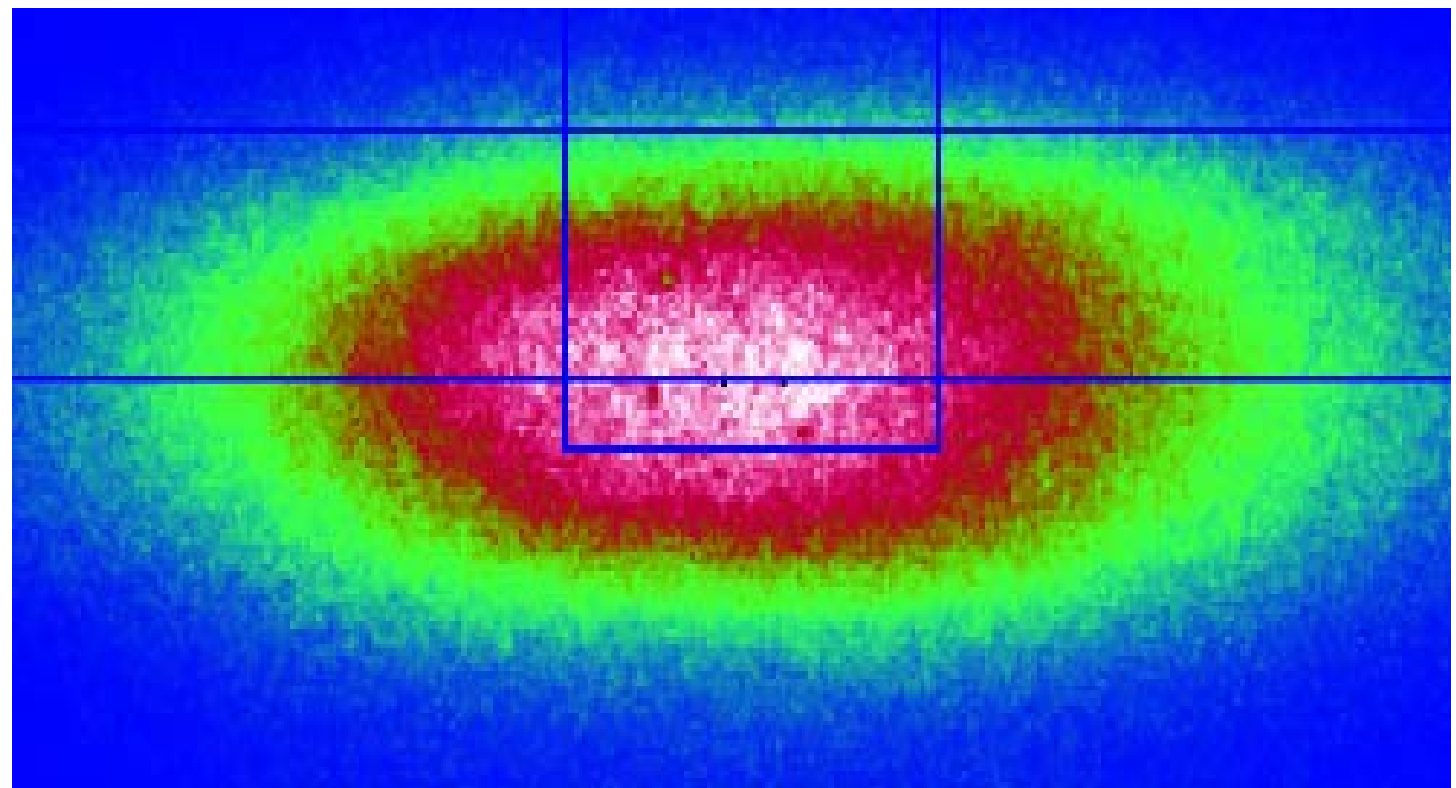

Figure 3: Transverse profile of a single bunch in LER using Bunch-by-bunch method, white shows highest intensity. 


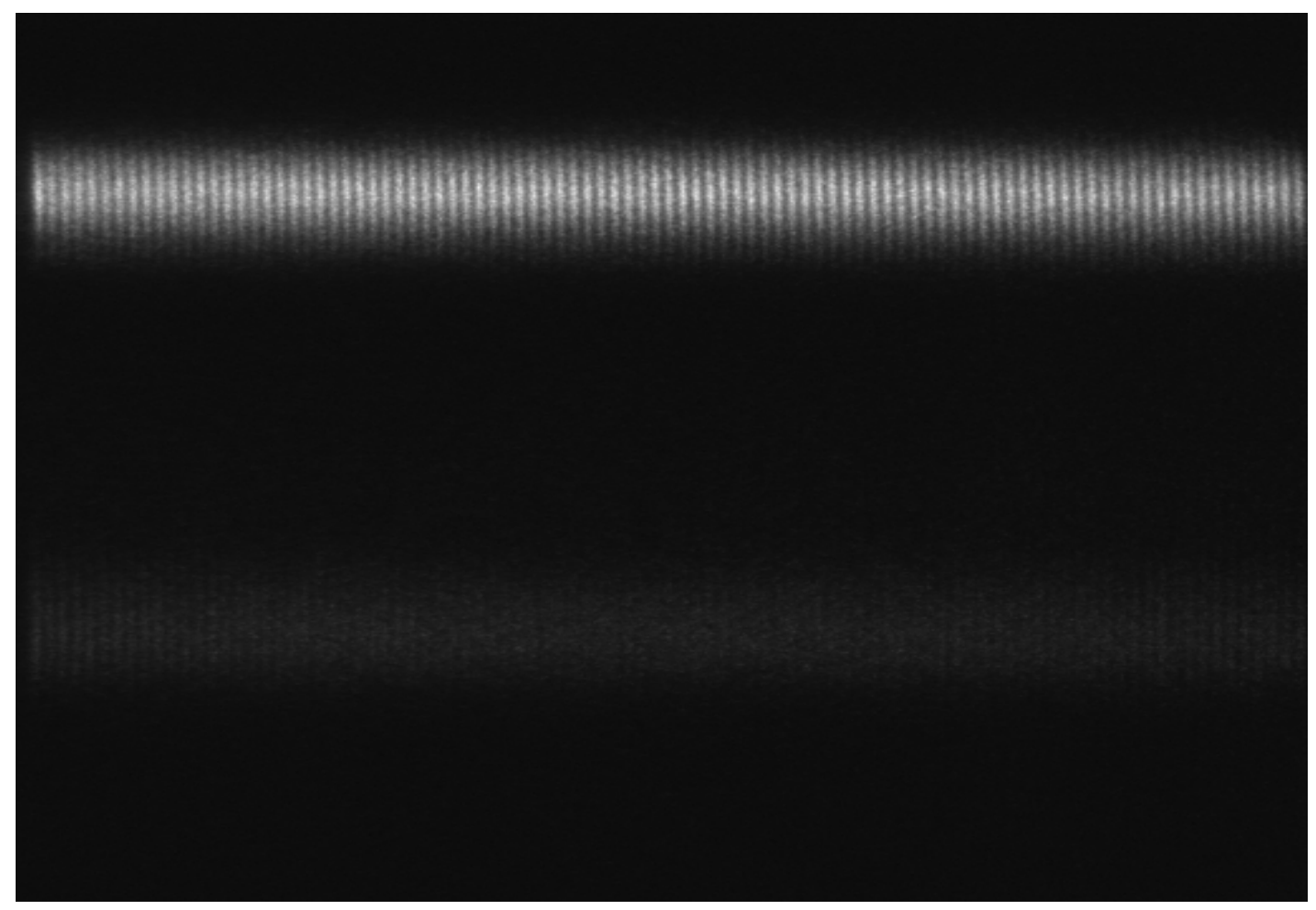

Figure 4: 94 turns of single bunch in LER, major axis shown above minor axis.

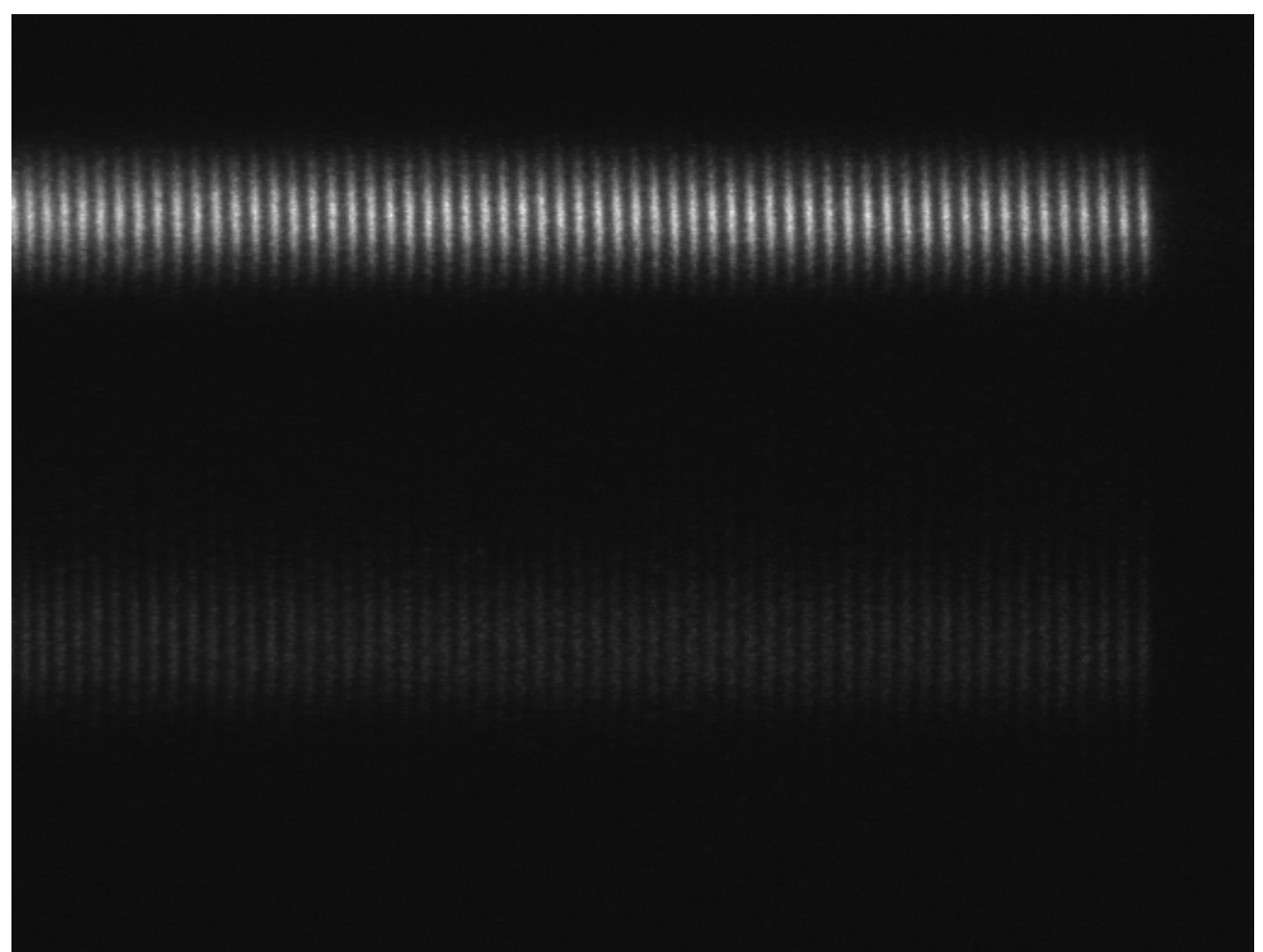

Figure 5: 60 turns of single bunch in LER, major axis shown above minor axis. 


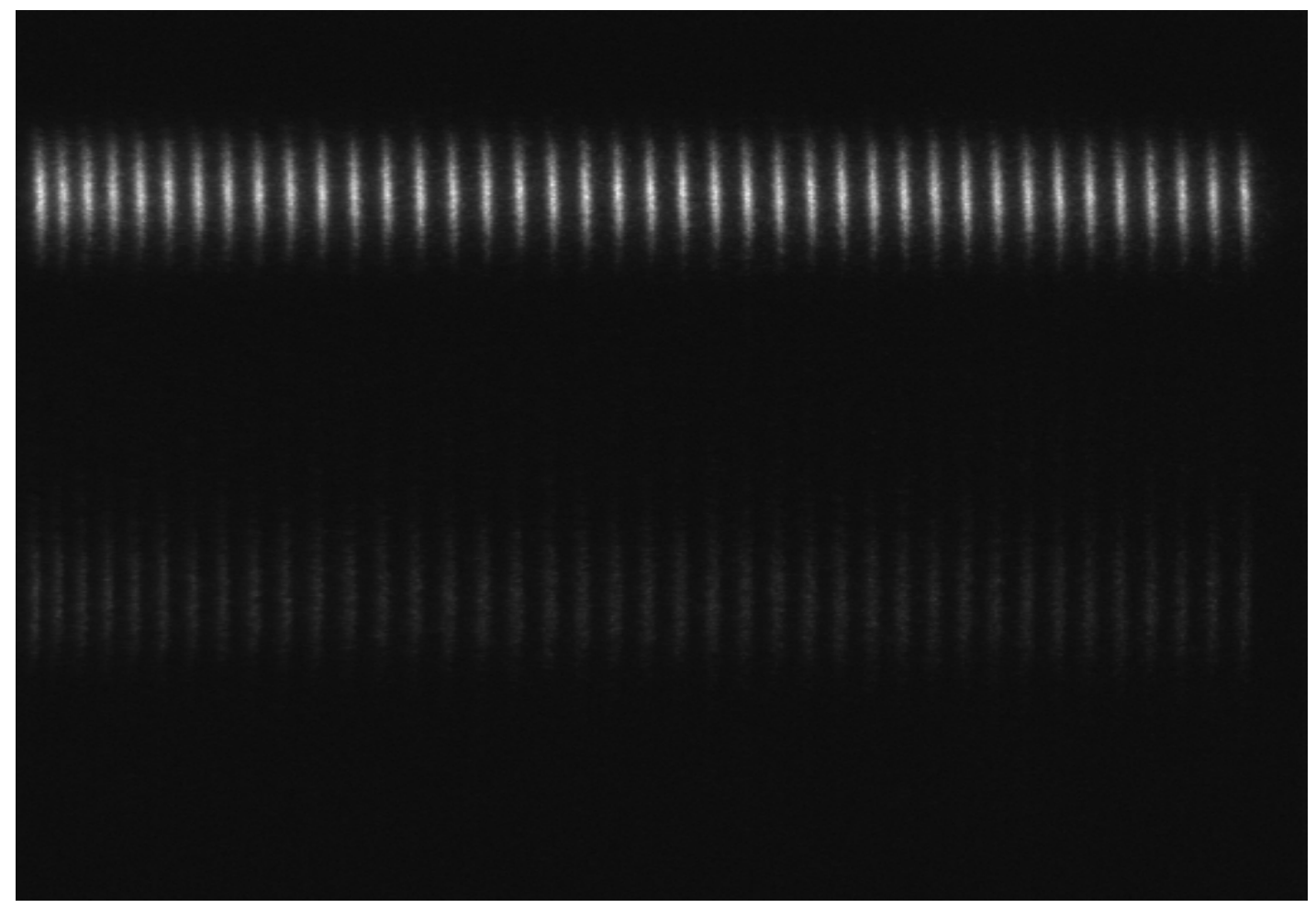

Figure 6: 41 turns of a single bunch in LER, major axis shown above minor axis.

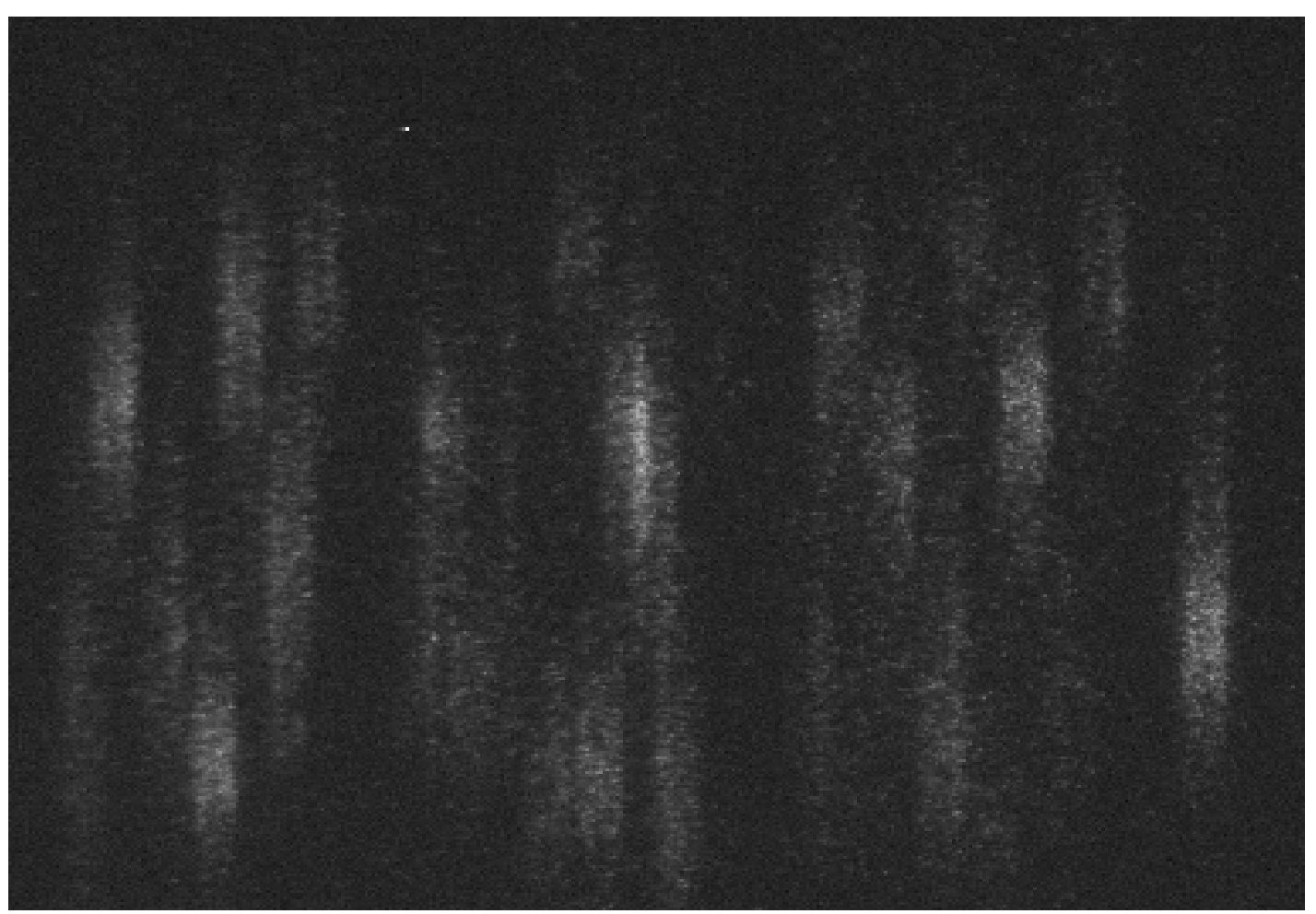

Figure 7: 50 turns of single injected bunch in LER, at beginning of ring fill. 


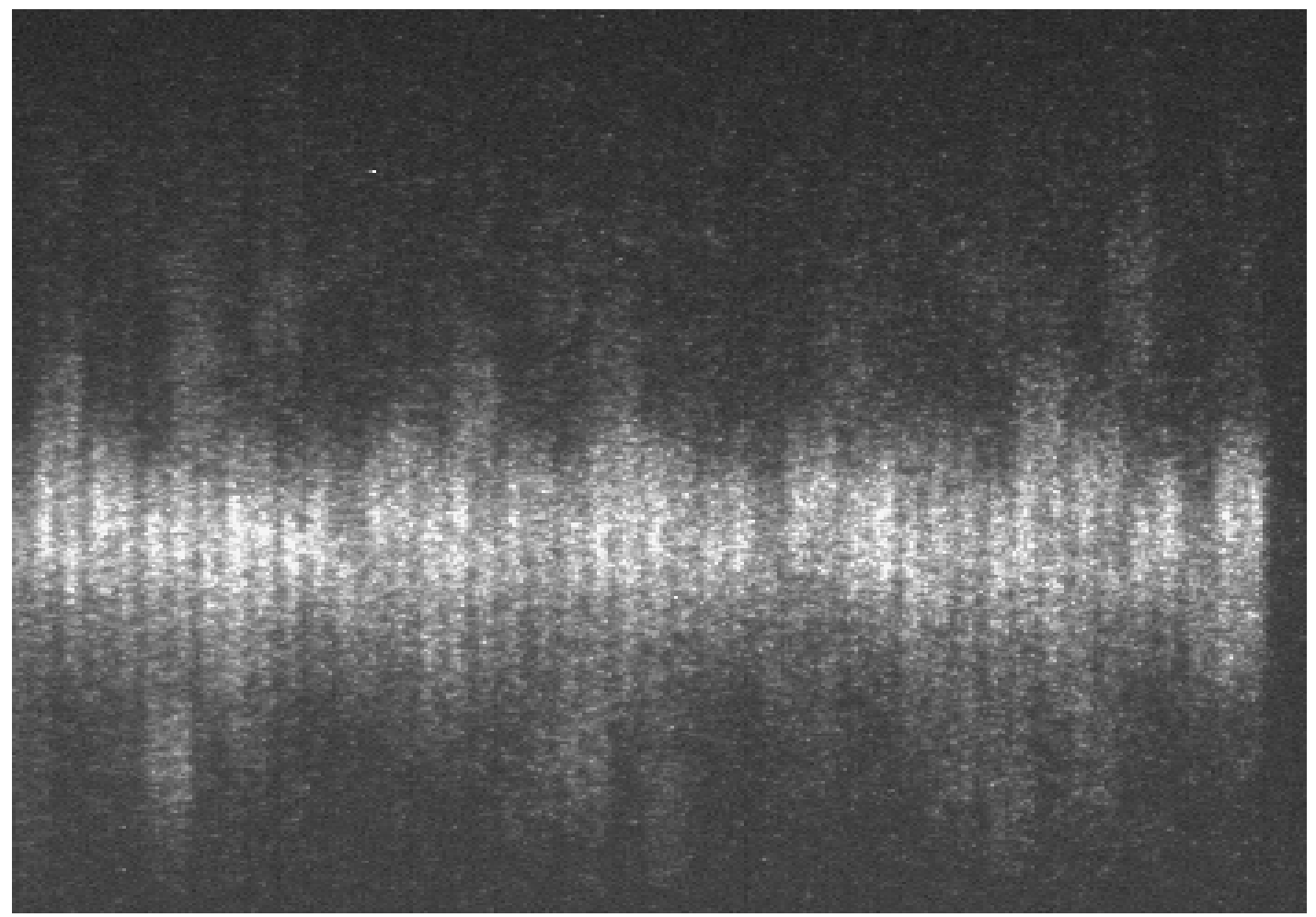

Figure 8: 50 turns of single injected bunch in LER oscillating around small stable bunch.

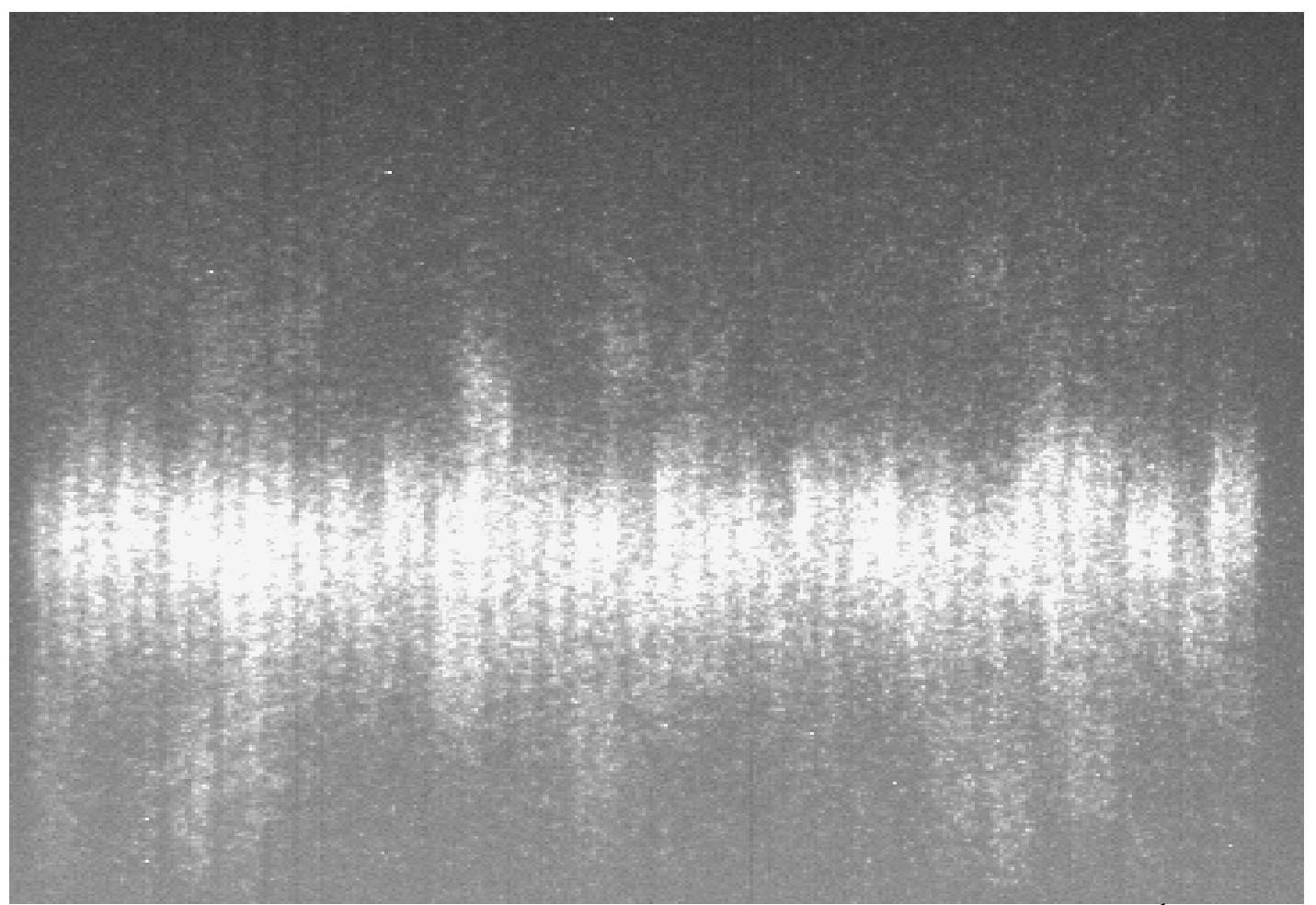

Figure 9: 50 turns of single injected bunch in LER oscillating around $1 / 3^{\text {rd }}$ full stable bunch. 